\title{
Drug Release Properties of Polymer Coated Ion-Exchange Resin Complexes: Experimental and Theoretical Evaluation
}

\author{
SEONG HOON JEONG, ${ }^{1}$ NAHOR HADDISH BERHANE, ${ }^{2}$ KAMYAR HAGHIGHI, $^{2}$ KINAM PARK $^{1}$ \\ ${ }^{1}$ Department of Pharmaceutics and Biomedical Engineering, Purdue University, West Lafayette, IN 47907 \\ ${ }^{2}$ Department of Agricultural and Biological Engineering, Purdue University, West Lafayette, IN 47907
}

Received 19 September 2005; revised 18 April 2006; accepted 21 April 2006

Published online in Wiley InterScience (www.interscience.wiley.com). DOI 10.1002/jps.20677

\begin{abstract}
Although ion-exchange resins have been used widely as drug delivery systems, their exact release kinetics has not been reported yet. Usually only the ratelimiting step has been taken into account and the rest of the steps have been ignored as instantaneous processes. To investigate the exact release kinetics of polymer-coated drug/ion-exchange resin complexes for sustained drug delivery, the results of new mathematical modeling were compared with experimental results. Drug/resin complexes with a model drug, dextromethorphan, were prepared and used as cores for fluid-bed coating. An aqueous colloidal dispersion of poly(vinyl acetate) was applied for the coating. A comprehensive mathematical model was developed using a mechanistic approach by considering diffusion, swelling, and ion-exchange processes solved by numerical techniques. The rate-limiting factor of the uncoated resin particles was diffusion through the core matrix. Similarly, in the coated particles the rate-limiting factor was diffusion through the coating membrane. The mathematical model has captured the phenomena observed during experimental evaluations and the release dynamics from uncoated and coated (at different coat levels) particles were predicted accurately (maximum RMSE 2.4\%). The mathematical model is a useful tool to theoretically evaluate the drug release properties from coated ion-exchange complexes thus can be used for design purposes. ( $) 2006$ Wiley-Liss, Inc. and the American Pharmacists Association J Pharm Sci 96:618-632, 2007
\end{abstract}

Keywords: ion-exchange resin; drug delivery; mathematical modeling; diffusion; fluid-bed coating

\section{INTRODUCTION}

Some of the early applications of ion-exchange resins were in the field of chemical engineering for purification of fluids from contaminants and separation of different gases. Since then, ionexchange resins have been used in the pharmaceutical industry as drug delivery systems. Their applications range from tablet disintegrants,

Correspondence to: Kinam Park, Professor (Telephone: 765494-7759; Fax: 765-496-1903;

E-mail: kpark@purdue.edu)

Journal of Pharmaceutical Sciences, Vol. 96, 618-632 (2007)

(C) 2006 Wiley-Liss, Inc. and the American Pharmacists Association taste-masking, and stabilization to key components of extended release formulations. ${ }^{1-3}$ Some drug products containing drug-loaded resins have been introduced in the market.

For the purpose of drug delivery, the drug release rate of ion-exchange resins can be modified depending on functional groups, ion exchange capacity, degrees of cross-linking, and particle size. However, the release rate from the resin complexes may not be satisfactory for sustained release. Thus, further control of the release rate has been achieved by applying a permeable coating. ${ }^{4-8}$ This implies that the drug release can be controlled by one or any combination of the core, coating and film diffusion resistances. 
Ion-exchange resins possess different release properties. They have fixed ionic functional groups which can provide binding of ionic drugs. Release of the bound drugs requires exchange with counter ions such as hydrogen or sodium, which are available in the gastrointestinal tract. The overall drug release kinetics of the polymer coated ionexchange resins are mainly dependent upon the drug and counter ion diffusion resistance in the coating film and the boundary layer (film) surrounding the particles. The dissociation of drug at the reaction surface is very fast as compared to other pertinent processes and barely does it affect the overall drug release kinetics.

To elucidate the drug release mechanisms and rate controlling steps in coated pellets or particles, considerable mathematical modeling efforts have been carried out for pharmaceutical ${ }^{9-16}$ as well as agricultural $^{17,18}$ applications. These studies deal with coated pellets with either granular soluble drug or matrix loaded with a soluble drug in the core. The processes involved in these systems are liquid diffusion, drug dissolution, and drug diffusion within the pellet and surrounding medium. In these studies, analytical derivations of the diffusion-dissolution equations were examined by applying simplifications and approximations. To make these equations amenable for analytical solving, usually only the process-limiting step is taken into account ignoring the dynamics of some pertinent processes. In fact, Frenning ${ }^{12}$ has recently reported that only a few consider dynamic descriptions of the pertinent processes as contributing steps to drug release from coated pellets. Moreover, these models can only be used for homogeneous dosage forms where the properties (notably diffusion coefficients) of the different domains (such as core and coating) are lumped which limit their applicability for design purposes. However, with the application of powerful numerical techniques, dynamic description of pertinent processes can be considered with less approximations and simplifications that can result not only in a more accurate prediction of the drug release profiles but also makes the models useful for design purposes such as selection of particle type and size, and coating type and thickness.

The objective of this study was to investigate drug release properties of polymer coated ionexchange resin complexes using experimental evaluation and to formulate a mechanistic drug release model by considering dynamic description of pertinent processes (diffusion ion-exchange, swelling) using numerical approach. It is believed that such modeling approach will lead to accurate prediction of the drug release by using physically meaningful model parameters instead of empirical ones. Such a tool is important for further understanding release mechanisms and deign purposes leading to renewed interest in the ion-exchange resin system for taste-masking and sustained drug release for new fast-melting tablet formulations of various ionic drugs.

\section{EXPERIMENTAL}

\section{Materials}

An ion-exchange resin (Dowex ${ }^{\circledR}$ 50WX4-200, polystyrene sulfonate, $\mathrm{H}^{+}$form, $4 \%$ divinylbenzene content) was purchased from Sigma-Aldrich (Sigma-Aldrich, Inc., St. Louis, MO). The resin particles were purified by rinsing $200 \mathrm{~g}$ of wet resin three times with $1000 \mathrm{~mL}$ of distilled water, twice with $95 \%$ ethanol, and then twice with $1000 \mathrm{~mL}$ of distilled water to remove the ethanol. Each treatment took at least $8 \mathrm{~h}$ by a batch process. After filtration, the resin was dried in a $45^{\circ} \mathrm{C}$ oven, and the moisture content was evaluated using a Karl Fischer titrator (Model 270, Denver Instrument, Arvada, CO). Dextromethorphan hydrobromide monohydrate (DM), a model drug, was obtained from Spectrum ${ }^{\circledR}$ (Spectrum Chemical Mfg. Corp., New Brunswick, NJ), and Kollicoat $\mathrm{SR}^{\mathrm{R}}$ 30D (polyvinyl acetate aqueous dispersion) was donated by BASF (BASF Corp., Mont Olive, NJ). Kollicoat ${ }^{\mathbb{R}}$ SR 30D is known as one of the aqueous colloidal polymer dispersions for the manufacturing of $\mathrm{pH}$-independent sustained release drug delivery systems. The polymer dispersion is based on polyvinyl acetate $(27 \%$ $\mathrm{w} / \mathrm{w})$ stabilized with polyvinylpyrrolidone $(2.7 \%$ $\mathrm{w} / \mathrm{w})$ and sodium lauryl sulfate $(0.3 \% \mathrm{w} / \mathrm{w})$.

\section{Preparation of DM-Loaded Resin Complex}

The DM-loaded Dowex ${ }^{\circledR}$ 50WX4-200 complex was prepared by a modified batch process. The purified resin particles were dispersed in a $1.9 \%$ $\mathrm{w} / \mathrm{v}$ drug solution with magnetic stirring at room temperature for $3 \mathrm{~h}$. After decanting the clear supernatant carefully, the same volume of fresh drug solution was added and stirred again for $5 \mathrm{~h}$ at room temperature. The complex was separated from the supernatant by vacuum filtration, washed with deionized water to remove any uncomplexed drugs, and then dried in the 
oven. The drug content of the resin complex was examined.

\section{Preparation of Polymer Coated Resin Complexes}

The DM-loaded resin complex was coated with Kollicoat $\mathrm{SR}^{\mathbb{R}}$ 30D in a fluidized-bed coater, MFL01 (Vector Corporation, Marion, IA) to obtain a predetermined weight gain. Bottom spray coating method (Wurster process) was applied for this process. The dried resin complex ( $40 \mathrm{~g}$ ) was mixed with micronized talc $(0.8 \mathrm{~g})$ to improve the initial flowability before the coating process. The coating solution was diluted to $10.0 \% \mathrm{w} / \mathrm{w}$ solid content. In order to enhance the film formation and the flexibility of the films, plasticizer (triethyl citrate) was added. Formulations of the coating solution and operating conditions of the fluid-bed coater are shown in Table 1 . The maximum amount of coating applied was $20 \% \mathrm{w} / \mathrm{w}$ as compared with the amount of core (resin complex).

\section{Drug Release Test}

Drug release tests from the uncoated and coated resin complex particles were conducted according to USP 27 Apparatus 2 guidelines (the paddle method) (Vankel ${ }^{\circledR}$ VK 7000, Vankel, Edison, NJ) with $900 \mathrm{~mL}$ dissolution medium maintained at $37 \pm 0.5^{\circ} \mathrm{C}$ and mixed at $100 \mathrm{rpm}$. The dissolution media used in this study were $0.1,0.01,0.001 \mathrm{~N}$ $\mathrm{HCl}$. Samples were withdrawn at predetermined time intervals and analyzed for drug content using HPLC system (Agilent 1100 Series, Agilent Technologies, Waldbronn, Germany) at a wavelength of $280 \mathrm{~nm}$. Samples were filtered with $0.2 \mu \mathrm{m}$ nylon filters, and then $20 \mu \mathrm{L}$ of the sample was injected. The column used for the analysis

Table 1. Formulations and Operating Conditions for the Preparation of Coated Particles

\begin{tabular}{lr}
\hline Conditions & Value \\
\hline Coating Solution & \\
Kollicoat SR ${ }^{\circledR}$ 30D $(\mathrm{g})$ & 70.00 \\
Triethyl Citrate (g) & 1.05 \\
Water (g) & 149.45 \\
Operating Conditions & \\
Inlet Air Flow $(\mathrm{l} / \mathrm{m})$ & 42.0 \\
Inlet Air Temperature $\left({ }^{\circ} \mathrm{C}\right)$ & 60.0 \\
Exhaust Temperature $\left({ }^{\circ} \mathrm{C}\right)$ & 24.3 \\
Nozzle Pressure $(\mathrm{psi})$ & 15.7 \\
Pump Speed (rpm) & 10.0 \\
\hline
\end{tabular}

was a Symmetry ${ }^{\circledR} \mathrm{C}_{18} 5 \mu \mathrm{m}(3.9 \times 150 \mathrm{~mm})$ (Waters Corporation, Milford, MA) with Sentry ${ }^{\mathrm{TM}}$ guard column (Symmetry ${ }^{\circledR} \mathrm{C}_{18} 5 \mu \mathrm{m}, 3.9 \times 20 \mathrm{~mm}$ ). The mobile phase contained a mixture of aqueous buffer $\left(10 \mathrm{mM} \quad \mathrm{KH}_{2} \mathrm{PO}_{4}\right.$ adjusted to $\mathrm{pH} 2.6$ with phosphoric acid) and acetonitrile in a volume ratio of 26:74. The retention time of $\mathrm{DM}$ was $3.1 \mathrm{~min}$ and every standard calibration curve was made before analysis to monitor the linearity from 0.5 to $250 \mu \mathrm{g} / \mathrm{mL}$.

\section{Determination of Diffusion Coefficients Through the Polymer Film}

DM permeation studies through the cast films were performed using side-by-side diffusion cells (PermeGear, Inc., Bethlehem, PA). Polymer films were prepared from coating solution by casting on a Teflon surface. The casting molds were prepared in house. The films were dried in an oven for $24 \mathrm{~h}$ at $60^{\circ} \mathrm{C}$ and then stored for $1 \mathrm{~h}$ at room temperature and $60 \%$ relative humidity. The dried films were peeled off the Teflon surface and cut into pieces with the size enough to cover the effective diffusion area of the cells. The thickness of the films was determined using a manual micrometer (Mitutoyo Corp., Kawasaki, Japan) with an accuracy of $\pm 1 \mu \mathrm{m}$ by measuring at least at seven random positions on the films. The cast films were placed between the two diffusion chambers. For the diffusion coefficient of DM through the film, one compartment was filled with $3.0 \mathrm{~mL}$ of DM-saturated solution with excess drug particles, and the other compartment was filled with $3.0 \mathrm{~mL}$ of $0.1 \mathrm{~N} \mathrm{HCl}$. For the diffusion coefficient of counter ion $\left(\mathrm{H}^{+}\right)$through the film, one compartment was filled with specific amount of drug loaded resin complex particles suspended in $3.0 \mathrm{~mL}$ water and the other compartment was filled with $3.0 \mathrm{~mL}$ of $0.1 \mathrm{~N}$ $\mathrm{HCl}$ solution. The exposed surface area was $0.636 \mathrm{~cm}^{2}$. The volume of each compartment was $3.0 \mathrm{~mL}$, and the temperature of the system was maintained at $37^{\circ} \mathrm{C}$ throughout the experiments. An equal volume of fresh buffer was immediately added to the side where sampling occurred. DM concentrations were quantified by HPLC analysis and corrected for dilution by sampling. The effect of compound withdrawal was taken into account when calculating the accumulated amount of the drug permeated. Polymer films with three different thicknesses were evaluated for this test. Diffusion coefficients $\left(\mathrm{cm}^{2} / \mathrm{s}\right)$ of DM and counter ion through the polymer can be obtained 
when plotting the flux and the concentration gradient and the values beyond the lag time were averaged. For the diffusion coefficient of the counter ion, it was assumed that bound drug especially in the surface of the particles could be released as soon as the counter ion passed through the membrane.

\section{Swelling Kinetics of Ion-Exchange Resins}

Measuring the volume change of swollen particles is a simple way to characterize the swelling kinetics and swelling equilibrium. Swelling kinetics of coated and uncoated resin complexes were measured using luer-lock and non-jacketed glass liquid chromatography column (size: $1.0 \mathrm{~cm} \times$ $20 \mathrm{~cm}$, bed volume $16 \mathrm{~mL}$, Sigma-Aldrich). A certain amount of the coated and uncoated resin complex particles was transferred and packed to the glass column equipped with a coarse frittedglass disk at the bottom. Aqueous medium was pumped upward using a peristaltic pump (Minipuls 2, Gilson, France) to wet all the particles at room temperature. Volume change of the particles in the column was monitored at predetermined time intervals and the swelling ratio $\left(R_{\mathrm{S}}\right)$ can be calculated as follows:

$$
R_{\mathrm{s}}=\frac{V_{\mathrm{s}}-V_{\mathrm{d}}}{V_{\mathrm{d}}}
$$

where $V_{\mathrm{s}}$ and $V_{\mathrm{d}}$ are the volume of swollen and dried resin particles, respectively.

\section{Scanning Electron Microscopy (SEM)}

Morphologies of DM-loaded resin complex and coated resin particles were examined by the SEM. Dried samples were attached to specimen stubs using double-sided copper tape and sputter coated with gold-palladium in the presence of argon gas using a Hummer I sputter coater (Anatech Ltd., Denver, NC). The samples were imaged with a JEOL JSM-840 scanning electron microscope (JEOL USA Inc., Peabody, MA) using $5 \mathrm{kV}$ accelerating voltage, $26 \sim 28 \mathrm{~mm}$ working distance, and probe current of $3 \times 10^{-11} \mathrm{amp}$.

\section{Confocal Laser Scanning Microscopy (CLSM)}

The internal structure of coated resin particles was imaged using an MRC-1024 Laser Scanning Confocal Imaging System (Bio-Rad Laboratories, Hercules, CA) equipped with a krypton/argon laser and a Nikon Diaphot 300 inverted micro- scope. All confocal fluorescence pictures were taken with a $20 \times$ objective. Nile Red, a lipophilic fluorescence dye, was added to the coating solution to visualize the coating film and the core appeared blue due to the self-fluorescence of the DM. Red and blue fluorescence images and transmission images were obtained from separate channels.

\section{THEORETICAL FRAMEWORK}

Several semi-empirical models for drug release from different drug delivery formulations exist. Among them the power models (Higuchi, ${ }^{19}$ Korsemeyer, ${ }^{20}$ and Peppas $^{21}$ ) and the Weibul models (Boyd, ${ }^{22}$ Reichenberg, ${ }^{23}$ and Bhaskar ${ }^{5}$ ) can be mentioned. A review of the different models is given in Costa and Sousa Lobo ${ }^{24}$ In the present study, the well-known model for ionexchange, the Boyd ${ }^{22}$ model which was later modified by Reichenberg ${ }^{23}$ and Bhaskar ${ }^{5}$ was employed to conduct preliminary study and to validate some of the assumptions of the detailed mathematical model. Then a more comprehensive numerical model was developed and validated.

\section{Boyd Models}

According to Boyd et al. ${ }^{22}$ the drug release from ion exchange resinate can be controlled by two kinds of diffusion processes, namely the diffusion of the drug across thin liquid film at the periphery termed as film diffusion and the diffusion of the drug in the matrix termed as particle diffusion. The rate-controlling step is either diffusion of drug across a thin liquid film at the periphery of the resin particle or diffusion of freed drug in a matrix. Both diffusion mechanisms are sequential steps so the slower one would be rate controlling. Assuming diffusion of a drug in the matrix is the rate-controlling step and all the resin particles are uniform spheres of radius $r$, the fraction of drug released, $F$, is given by the following expression. ${ }^{22}$

$$
F=\frac{M_{t}}{M_{\infty}}=1-\frac{6}{\pi^{2}} \sum_{n=1}^{\infty} \frac{e^{-n^{2} B t}}{n^{2}} \quad \text { where } B=\pi^{2} D_{i} / r^{2}
$$

$M_{t}$ and $M_{\infty}$ are the amounts of drug released after time $t$ and after infinite time, respectively. $B$ is the rate constant, $D_{i}$ represents the effective diffusion coefficient of the exchanging ions inside the resin particle, and $n$ is the summation variable. Since 
using Eq. (2) is not convenient due to its infinite terms, some simple approximations were sought such as that by Reichenberg ${ }^{23}$ (Eq. (3)).

$$
B t=\left\{\begin{array}{l}
2 \pi\left(\frac{1-\pi}{6} F-\left(1-\frac{\pi}{3} F\right)^{0.5}\right) \text { for } F \leq 0.85 \\
-\ln (1-F)-0.04977 \text { for } F>0.85
\end{array}\right.
$$

If the plot of the $B t$ corresponding to the $F$ against time gives a straight line with a slope equal to $B$, it can be assumed that drug diffusion within the resin matrix is the rate-controlling step and the diffusivity can be obtained using the $B$ value. $^{4,6,8,25}$ Bhaskar proposed a more elegant approximation based on the monotonic transform method:

$$
-\ln (1-F)=1.59\left(\frac{6}{r}\right)^{1.3}\left(D_{i} t\right)^{0.65}
$$

Hence, particle diffusion control can be tested by the linearity between $-\ln (1-F)$ and $t^{0.65}$ with the slope being a quantity related to the diffusivity and particle radius.

For film-diffusion controlled process Boyd et al. ${ }^{22}$ proposed a model based on the assumption that the film thickness and distribution coefficient are constant in each resinate particle.

$$
-\ln (1-F)=\frac{3 P t}{r}
$$

where $P$ is the apparent permeability of the film. The plot $-\ln (1-F)$ versus $t$ provides a linear line with a slope related to the apparent permeability, $P$.

\section{Mechanistic Model}

The processes involved during the release phase are: (i) diffusion of the counter ion through the boundary layer, coating, and resin complex, (ii) dissociation (ion exchange reaction), and (iii) diffusion of freed drug through the core and/or coating. Since the drug release kinetics of the delivery system is directly correlated with the functionality of individual particles, it is a common practice to consider a single particle for the mathematical treatment. The mathematical model was formulated based on the following assumptions. (i) The effects of convection and pressure gradients can be ignored. (ii) The pellets were considered spherical in shape, based on the SEM and CLSM images shown in Figures 1A, 3 and 4. (iii) The boundary layer on the surface of the coating was ignored due to the hydrophilic nature of the drug and existence of vigorous mixing, and a perfect sink condition with equilibrium counter ion concentration on the surface was considered. Note that this assumption rules out the possibility of film diffusion as a controlling mechanism a priori which should be confirmed from the preliminary investigation using simpler models. (iv) The pellets were assumed to be homogeneous and isotropic. As a result of this and the foregoing perfect sphere assumption, a symmetry condition was invoked resulting in a 2-D model of a single pellet. One quarter of the geometric model of a typical pellet as considered for the simulation is shown in Figure 1B.

Given these assumptions the processes can generally be described by the reaction-transport equation.

$$
\frac{\partial c_{i}}{\partial t}=\nabla J_{i}+R x
$$

Where $c$ is the concentration of the diffusing species in the pellet and the subscript $i$ denotes the species $(i=1$ for the water, $i=2$ for the counter ion and $i=3$ for the drug); $t$, the time; $J_{i}$, the net flux; and $R x$, the reaction source term.
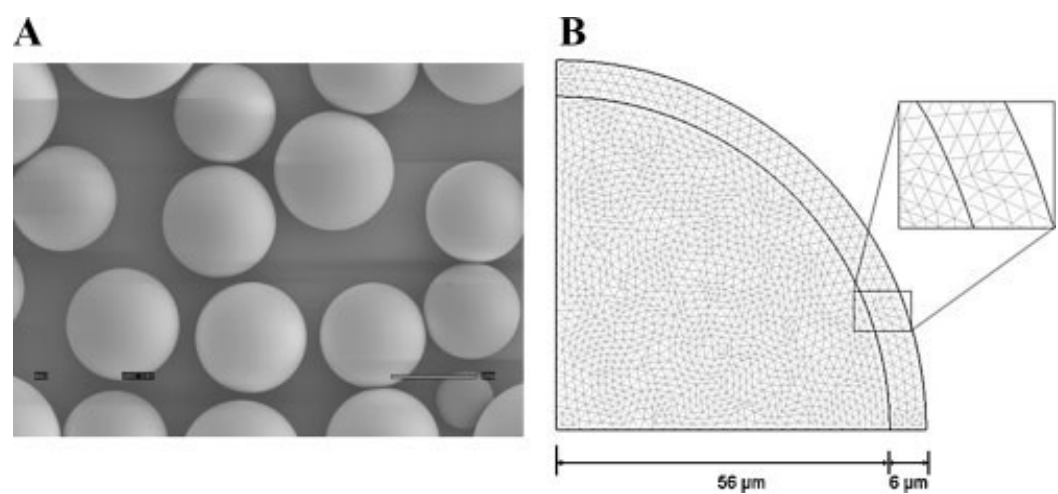

Figure 1. Scanning electron micrograph of DM-Dowex ${ }^{\circledR} 50 \mathrm{WX} 4-200$ resin particles (A) and a schematic diagram showing the geometric model of a quarter polymer coated resin complex (B). 
The most important feature that distinguishes ion exchange from isotopic exchange is the electric coupling of the ionic fluxes. This means the diffusion process is governed by the Brownian motion and the effect of the electric charge on the movement of the ions. Here, it should be noted that the water does not take part in the electric coupling of the ions and is considered here for the effect of its (water) ingress on the swelling of the pellets. The resulting flux of the species $(i=2,3)$ can be described by the Nernst-Plank equation. ${ }^{26}$

$$
\begin{aligned}
J_{i} & =\left(J_{i}\right)_{\text {diff }}+\left(J_{i}\right)_{\mathrm{el}} \\
& =-D_{i}\left(\nabla c_{i}+z_{i} c_{i} \frac{F}{R T} \nabla \vartheta\right) ; i=2,3
\end{aligned}
$$

where $D$ is the diffusion coefficient; $z_{i}$, the valence; $F$, Faraday constant; $R$, gas constant; $T$, absolute temperature; $\vartheta$, electric potential.

Assuming that there are no co-ions present and the swelling of the resin is neglected, the restrictions of electro-neutrality $\left(\sum z_{i} c_{i}=\right.$ const $)$ and absence of electric current $\sum z_{i} J_{i}=0$ apply. Applying these constrains on Eq. 7 yields:

$$
J_{i}=D_{\mathrm{DI}} \nabla c_{i}
$$

with

$$
D_{\mathrm{DI}}=\frac{\left(\prod D_{i}\right)\left(\sum z_{i} c_{i}\right)}{\sum z_{i}^{2} D_{i} c_{i}}=\frac{\left(\sum z_{i} c_{i}\right)}{\sum z_{i}^{2} \frac{c_{i}}{D_{i}}}
$$

The reaction source term $R x$ can be described as:

$$
R x=k \prod_{n} c_{i}
$$

where the subscript $n$ represents the reactant species, in this case, the counter ion and the drugresin complex; and $k$ is the reaction rate constant. The physical interpretation of the reaction constant has little in common with the rate constants in the actual chemical reaction; rather it is a partition coefficient describing how the ions are partitioned between the phases. Moreover, the reaction term is constrained by the availability of the resin-drug complex and once depleted the term disappears. Due to the speed of the dissociation, chemically controlled process was excluded unequivocally as a rate controlling mechanism. $^{22,26}$ Thus, the reaction term can be ignored and included here only for the sake of completeness. Note that these equations only apply to the core matrix. For the coating, only Fickian diffusion occurs and the reaction source term does not apply.
To account for the swelling of the core of the pellet a moving boundary problem was considered using the Arbitrary Lagrangian-Eurelian (ALE) approach. After defining the initial domain $\Omega\left(x_{j}\right)$ and the moving domain $\hat{\Omega}\left(\hat{x}_{j}\right)$, Eq. 6 after substitution of Eq. 7 can be rewritten using the weak formulation in the deformed coordinate system as:

$$
\begin{aligned}
\int_{\hat{\Omega}} c_{i}^{t} \frac{\partial c_{i}}{\partial t} d \hat{\Omega}= & \int_{\hat{\Omega}}\left\{-D_{\mathrm{ID}} \sum_{j} c_{i, \hat{x}_{j}}^{t} c_{i, \hat{x}_{j}}+c_{i}^{t} R x_{i}\right\} d \hat{\Omega} \\
& +\int_{\partial \hat{\Omega}} D_{\mathrm{ID}} c_{i}^{t} \nabla c_{i} \cdot n d \hat{s}
\end{aligned}
$$

where $c^{t}$ is the test function, $n$ unit normal vector and $c_{i, x_{j}}$, the spatial derivatives of the concentration of the species. The movement of the mesh (moving domain) (or swelling) was described by the Poisson equation expressed in the weak form in the moving domain as:

$$
\int_{\hat{\Omega}} u_{\hat{x}_{j}}^{t} \cdot u_{\hat{x}_{j}} d \hat{\Omega}=0
$$

where $u$ is the prescribed deformation obtained from the swelling kinetics of the pellet resinate given by:

$$
u=\kappa c_{1}
$$

where $\kappa$ is the swelling constant.

The mapping between the original fixed domain and the deforming domain can be derived using the chain rule, which results in the Jacobian, ऽ:

$$
\Im=\frac{d \hat{x}_{j}}{d x_{j}}
$$

The mapping of the variables between the two domains can be expressed as:

$$
\begin{aligned}
c_{i, x_{j}} & =\Im^{-1} c_{i, \hat{x}_{j}} ; c_{i, x_{j}}^{t}=\Im^{-1} c_{i, \hat{x}_{j}}^{t} ; \\
u_{x_{j}} & =\Im^{-1} u_{\hat{x}_{j}} ; u_{i, x_{j}}^{t}=\Im^{-1} u_{i, \hat{x}_{j}}^{t} \text { and } d \hat{\Omega} \\
& =\operatorname{det}(\Im) d \Omega
\end{aligned}
$$

where $\operatorname{det}(\Im)$ is the determinant of the Jacobian.

The effect of the swelling on the diffusion coefficients was described using a Fujita-type exponential dependence ${ }^{27}$ expressed as:

$$
D_{i}=D_{i, \text { eq }} \exp \left(-\beta_{i}\left(1-\frac{c_{1}}{c_{1, \mathrm{eq}}}\right)\right)
$$

where $\beta_{i}$ and $D_{i \text {,eq }}$ are dimensionless constants 
and diffusion coefficients at the equilibrium swollen state respectively.

Since the core matrix is not hydrated, it was assumed that initially there was no free drug and no counter ions in the pellet. At the boundary (outer coating), the counter ion concentration was considered as the equilibrium concentration and a perfect sink condition for the drug. These considerations result in the following initial and boundary conditions for the diffusing species.

$$
\begin{gathered}
c_{i}(t=0, \forall r)=0 \\
c_{1}(t>0, r=R)=c_{1, \mathrm{eq}} \\
c_{2}(t>0, r=R)=0 \\
x_{j}(t=0)=\hat{x}_{j}
\end{gathered}
$$

The amount of released drug was calculated according to:

$$
W=\int_{\mathrm{d} s} J \cdot n \mathrm{~d} s
$$

where $W$ is the average release rate and $n$, the normal vector at the boundary. The amount of released drug $M_{t}$ is then given by:

$$
M_{t}=\int_{\mathrm{d} t} W(t) \mathrm{d} t
$$

The fractional drug release $M$ can then be calculated from:

$$
F=\frac{M_{t}}{M_{\infty}}
$$

where $M_{\infty}$ is the amount of release after a long time.

\section{RESULTS AND DISCUSSION}

\section{Effect of Kollicoat ${ }^{\circledR}$ SR 30D Coating Level on the Drug Release}

The amount of DM loaded in $100 \mathrm{mg}$ resin complex was $65.19 \mathrm{mg}( \pm 0.51)$. The DM release profiles of the coated uncoated resin particles are shown in Figure 2. The percentage level of coating was determined by the weight increase relative to the amount of core. The drug release decreased as the coating level increased and as a result the drug release rate could be modified easily by using different levels of the coating. Table 2 shows the

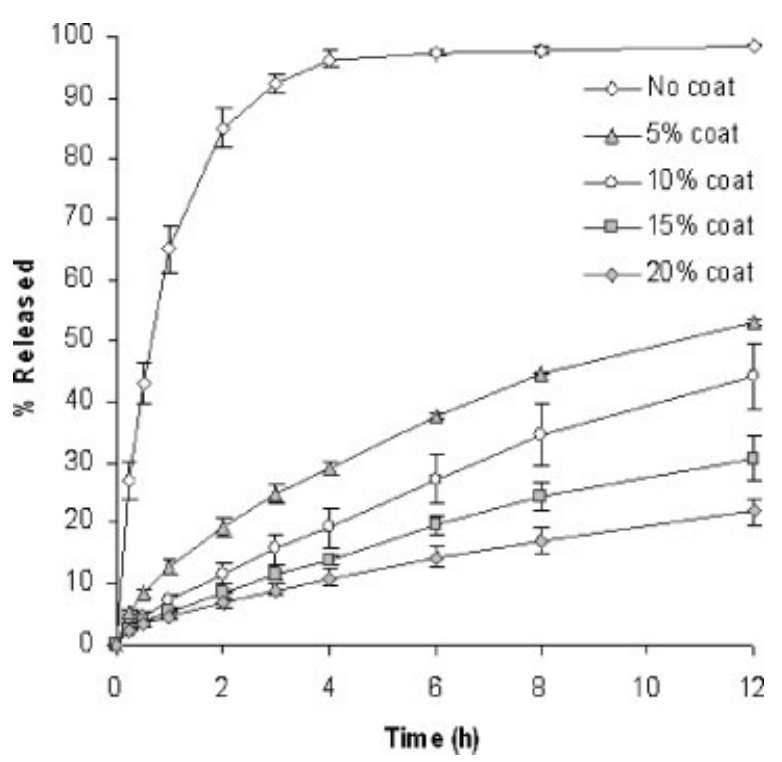

Figure 2. In-vitro release of DM from different levels of Kollicoat ${ }^{\mathbb{R}}$ SR 30D coating using cores of DM/Dowex ${ }^{\circledR}$ 50WX4-200 resin particles in simulated gastric fluid $(\mathrm{pH}=1.2)$ at $37^{\circ} \mathrm{C}$. The $5 \%$ coat indicates that the amount of coating is $5.0 \% \mathrm{w} / \mathrm{w}$ relative to the amount of core (drug/resin complex). Each point represent the mean \pm standard error $(n=3)$.

average particle size and coating thickness at different coating levels.

When DM-loaded resin particles were coated with Kollicoat ${ }^{\circledR}$ SR 30D, the release rates of DM from the particles decreased significantly even with only $5 \%$ coating. This large decrease can mainly be attributed to the large difference in diffusion coefficient of between the two materials. Due to the low cross-linking of the uncoated resin particles, swelling takes place as they come in contact with aqueous environments such as water or dissolution medium. This would result in increased effective surface area and also change

Table 2. Average Particle Size of Coated and Uncoated Ion-Exchange Resin Complexes and Their Coating Thickness at Different Coating Levels

\begin{tabular}{lcc}
\hline $\begin{array}{l}\text { Weight } \\
\text { Gain } \\
(\%)\end{array}$ & $\begin{array}{c}\text { Average Coated } \\
\text { Particle Diameter } \\
(\mu \mathrm{m})\end{array}$ & $\begin{array}{c}\text { Estimated Coating } \\
\text { Thickness } \\
(\mu \mathrm{m})\end{array}$ \\
\hline 0 & $106.2( \pm 27.7)$ & - \\
5 & $115.6( \pm 24.5)$ & 4.7 \\
10 & $122.7( \pm 17.3)$ & 8.3 \\
15 & $131.3( \pm 21.0)$ & 12.6 \\
20 & $138.3( \pm 25.1)$ & 16.1 \\
\hline
\end{tabular}


the effective diffusion coefficient of the resin. However, the presence of the coating constrains the swelling of the resin core which also contributed to the observed difference.

The films of Kollicoat ${ }^{\mathbb{R}}$ SR 30D polymer without plasticizers were slightly brittle in dry state. However, when wet, they were flexible enough to be elongated more than $100 \%$ so the crack formation on the surface of coating due to the swelling of the core would be prevented. This was confirmed by microscopic observation discussed in the following section. It was observed that when $5 \%$ of triethyl citrate (plasticizer) was used the flexibility of the polymer significantly increased, yielding an elongation value of more than $200 \%$.

\section{Microscopic Observation of the Coating}

\section{Scanning Electron Microscopy (SEM)}

As shown in Figure 3A, only 5\% coating covered all areas of the resin particles without any cracks and uncoated areas. As the level of coating increased (Fig. 3B-D), the coat looked thicker based on the surface morphology of the coated particles. The shape of the coated particles looked like a planet with many craters offering a nonuniformly coated surface. Several factors such as particle-particle collisions during the fluid-bed coating process, stresses that develop due to gravitational forces and surface tension gradients during drying of the coating (Marangoni instability) might have contributed to the observed nonuniformity. This affects the release profiles due to the shorter or longer distances the drug molecules travel through the non-uniformly coated area in the films.

\section{Confocal Laser Scanning Microscopy (CLSM)}

The coated resin particles with different levels of coating were compared with respect to their internal structures using the CLSM, as shown in Figure $4 \mathrm{~A}-\mathrm{D}$. In this experiment, the coated polymer membrane was visualized with Nile Red, a lipophilic fluorescence dye, and the core appeared blue due to the self-fluorescence of the DM. The pictures show that with the coating level increase the intensity and thickness of the red color increased. However, there are many black spots throughout the coating membrane showing that the coating is not homogeneous and has some defects. This confirms that there exist irregularities and anomalies in the coating, partially observed in the SEM pictures.

These SEM pictures and CLSM images provided valuable information for the development of the geometric model for the mathematical simulation in determining accurately the particle size and film thickness. Moreover, the observed
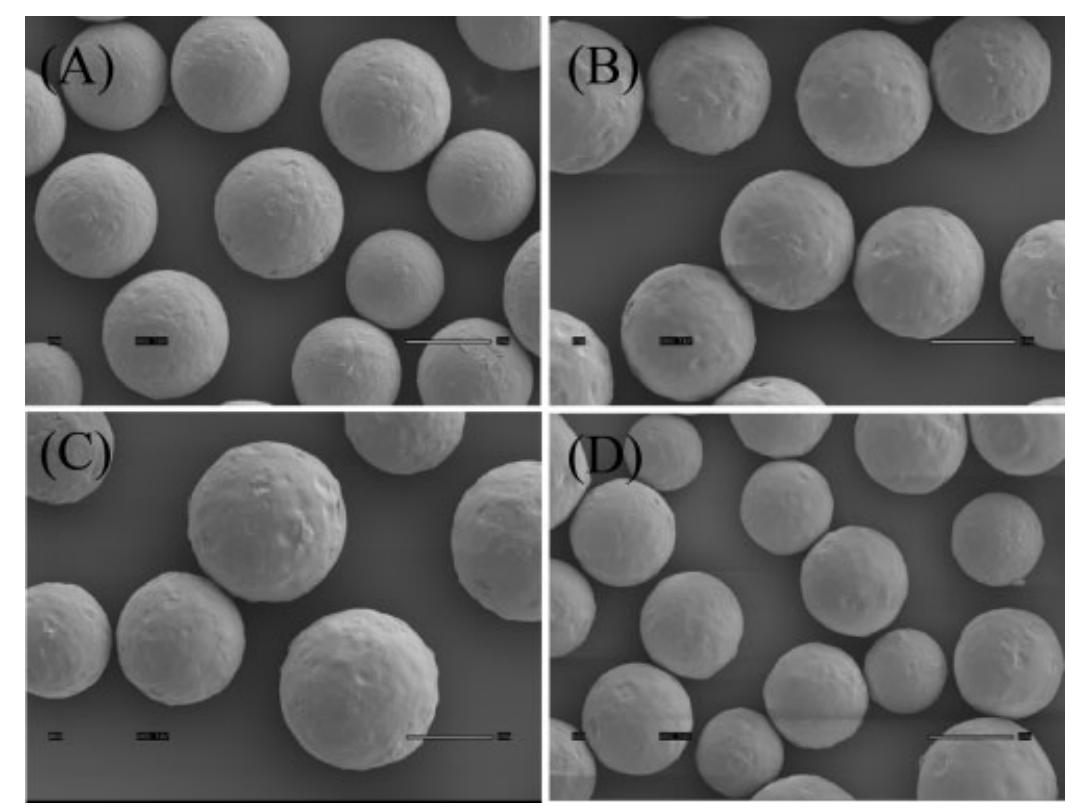

Figure 3. Scanning electron micrographs of Kollicoat ${ }^{\mathbb{R}}$ SR 30D coated DM-Dowex ${ }^{\circledR}$ 50WX4-200 resin particles, and the levels of coatings are 5\% (A), 10\% (B), 15\% (C), and $20 \%$ (D). 

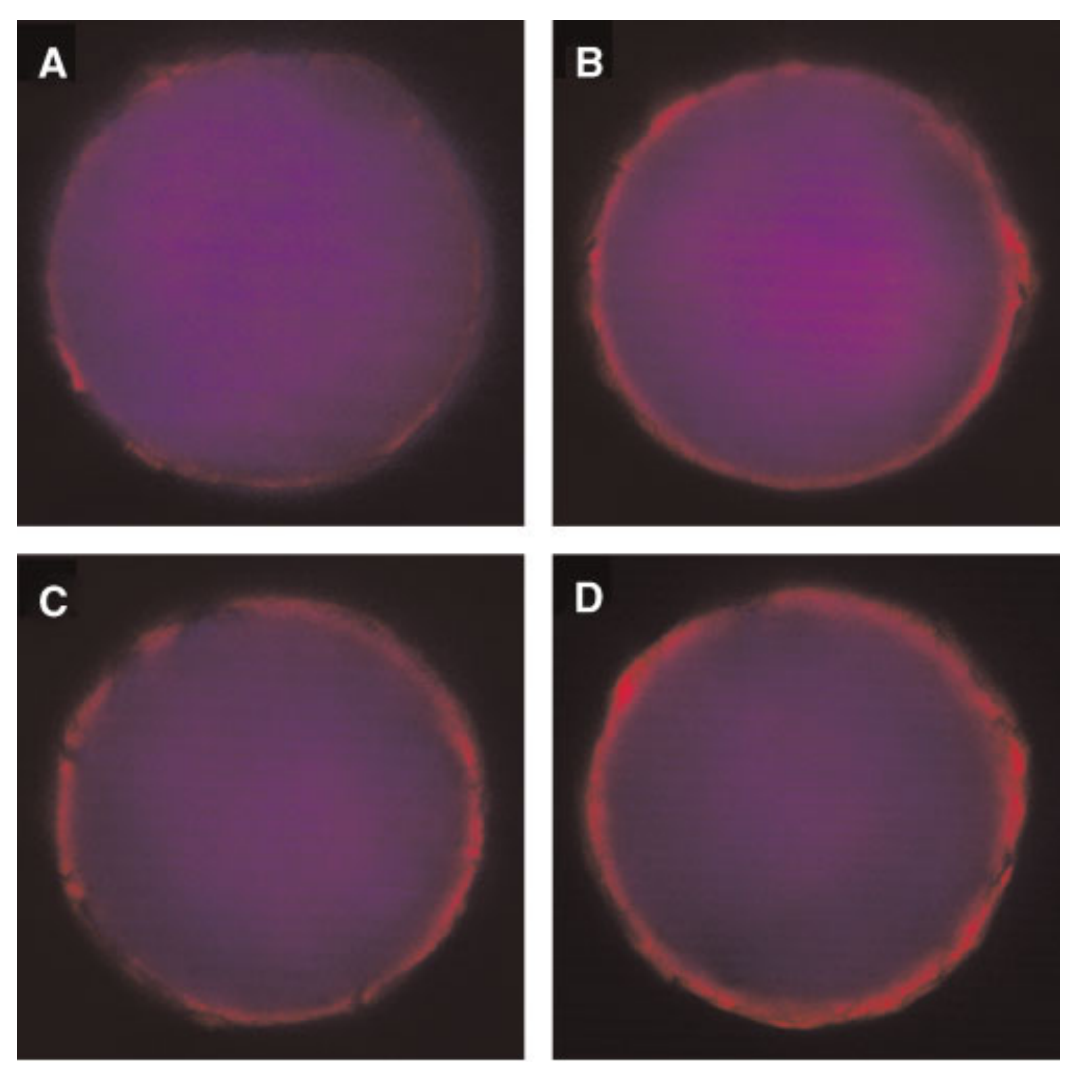

Figure 4. Confocal laser scanning microscopic images of Kollicoat ${ }^{\circledR}$ SR 30D coated DM-Dowex ${ }^{\circledR} 50 \mathrm{WX} 4-200$ resin particles, and the levels of coatings are $5 \%$ (A), 10\% (B), $15 \%$ (C), and 20\% (D). The polymer coating appears red due to the presence of Nile Red. The core appears blue due to the fluorescence of the drug. [Color figure can be seen in the online version of this article, available on the website, www.interscience.wiley.com.]

inhomogeneity and defects of the coating observed from these images helped diagnose the source of possible discrepancy between model and experimental data. This observation has lead to further theoretical investigation of the effects of these anomalies on the drug release.

\section{Drug Release Kinetics}

\section{Boyd Models}

In Figure 5A and B, the DM release profiles from the uncoated pellets were entered into the particle diffusion (Fig. 5A) and film diffusion (Fig. 5B) controlled Boyd models. The linearity obtained using the particle diffusion controlled is higher $\left(R^{2}=0.997\right)$ than that of the film diffusion controlled $\left(R^{2}=0.904\right)$. This suggested that drug diffusion in the resinate matrix of the uncoated pellets was the rate-controlling step. The presence of agitation can reduce the film layer thickness substantially decreasing the resistance thus favoring the particle diffusion as rate controlling mechanism. It should be noted, however, that due to the presence of ionic forces, strongly adhered ions on the surface of the pellet always remain unaffected by the presence of agitation providing a resistance layer. From the slope of the fitting curve, the diffusion coefficient of the resinate matrix was calculated and found to be $3.4 \times 10^{-10} \mathrm{~cm}^{2} / \mathrm{s}$ (Tab. 3). This value, even though slightly higher, is in the same order of magnitude as the value reported in literature for the same system (resinate) ${ }^{28}\left(1.24 \times 10^{-10} \mathrm{~cm}^{2} / \mathrm{s}\right)$.

In Figure 6A and B DM release profiles form coated pellets (different coating levels) were entered into the particle diffusion (Fig. 6A) and film diffusion (Fig. 6B) controlled Boyd models. Once more, for all the coating levels, the particle diffusion gives better linearity indicating that it is the rate controlling mechanism as observed from the correlation coefficients. It should be noted here that the model does not differentiate between the resin matrix and coating domains. Thus, the 

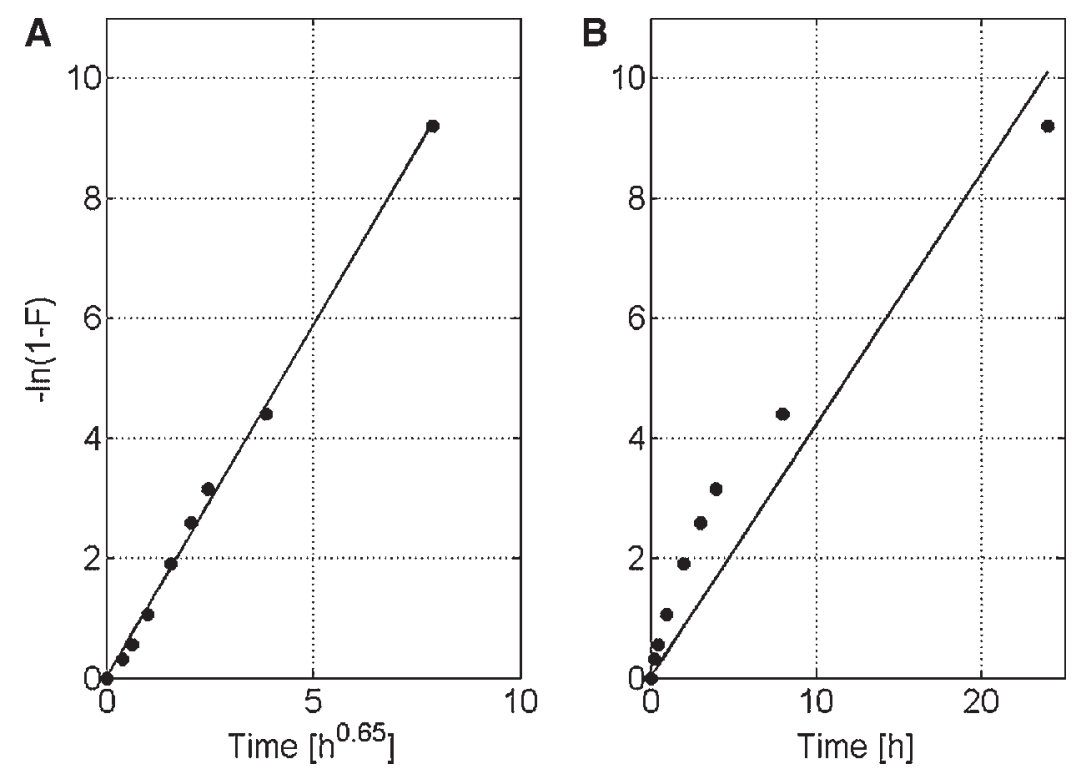

Figure 5. DM release profiles from uncoated pellets plugged into particle diffusion controlled model ( $\left.R^{2}=0.997\right)(\mathrm{A})$ and film diffusion controlled model $\left(R^{2}=0.904\right)(\mathrm{B})$.

diffusion coefficient calculated from the slopes of the coated pellets result in fictitious (lumped) value that is the resultant of the diffusivities of the coating and the core polymers which, in the present case, differ by two orders of magnitude. It was observed that the slopes of the plots were strongly dependent on the coating level. Therefore, increasing the coating thickness by applying more amount of polymer dispersion would be one way to reduce the diffusion rate through the coating. This, in turn, suggests that the particle diffusion through the coating polymer is particularly the rate controlling mechanism. However, one of the limitations is that one can not use these models for design purposes with respect to the type of resin, particle size, type and thickness of the coating polymer due to the fact that these domains (core and coating) are treated as a homogeneous system.

From the foregoing results, it is apparent that the film diffusion plays an insignificant role in controlling the rate of release of DM from the ionexchange pellets. However, to delineate the boundary between the two rate processes and determine the scope of the models with respect to the environmental condition of the medium, further investigation was conducted. Boyd et al. ${ }^{22}$ argue that in the case where ionic concentration of the ingoing ion in the release medium is small, the film diffusion becomes the rate controlling mechanism over the particle diffusion. In

Table 3. Model Parameters

\begin{tabular}{llc}
\hline Symbol/Unit & \multicolumn{1}{c}{ Description } & Value \\
\hline$\beta_{1=2=3}$ & Dimensionless Constant & -0.31 \\
$D_{11}\left(\mathrm{~cm}^{2} / \mathrm{s}\right)^{b}$ & Diffusion Coefficient of Water in the Core & $4.3 \times 10^{-6}$ \\
$D_{21}\left(\mathrm{~cm}^{2} \mathrm{~s}^{-1}\right)^{b}$ & Diffusion Coefficient of Counter Ion in the Core & $4.3 \times 10^{-6}$ \\
$D_{22}\left(\mathrm{~cm}^{2} \mathrm{~s}^{-1}\right)^{c}$ & Diffusion Coefficient of Counter Ion in the Coating & $1.0 \times 10^{-7}$ \\
$D_{31}\left(\mathrm{~cm}^{2} \mathrm{~s}^{-1}\right)^{a}$ & Diffusion Coefficient of Drug in the Core & $(3.4 \pm 1.1) \times 10^{-10}$ \\
$D_{32}\left(\mathrm{~cm}^{2} \mathrm{~s}^{-1}\right)^{c}$ & Diffusion Coefficient of Drug in the Coating & $(4.0 \pm 1.3) \times 10^{-12}$ \\
$c_{1, \text { eq }}\left(\mathrm{g} \mathrm{cm}^{-3}\right)^{d}$ & Equilibrium Concentration of Counter Ion & 0.85 \\
$\mathrm{M}_{0}(\mathrm{~g})^{c}$ & Initial Drug Load & $5.09 \times 10^{-7}$ \\
\hline
\end{tabular}

${ }^{a}$ Estimated indirectly from measured data.

${ }^{b}$ Adopted from Ref. ${ }^{29}$

${ }^{c}$ Measured.

${ }^{d}$ From manufacturer manual. 

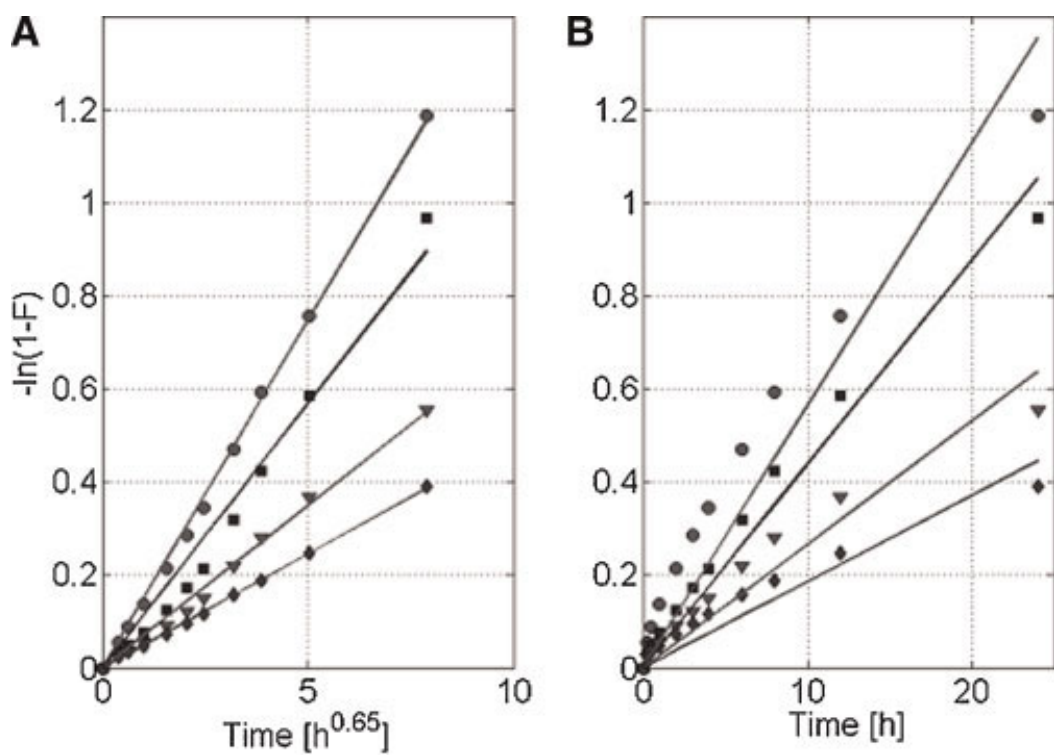

Figure 6. DM release profiles from coated pellets (different degree of coating) plugged into particle diffusion controlled model $0: 5 \% \mathrm{w} / \mathrm{w}$ coating $\left(R^{2}=0.999\right)$; $\square: 10 \% \mathrm{w} / \mathrm{w}$ coating $\left(R^{2}=0.977\right) ; \nabla: 15 \%$ w/w coating $\left(R^{2}=0.993\right) ; \diamond: 20 \%$ w/w coating $\left(R^{2}=0.999\right)$ (A); and film diffusion controlled model0: $5 \% \mathrm{w} / \mathrm{w}$ coating $\left(R^{2}=0.90\right)$; $: 10 \% \mathrm{w} / \mathrm{w}$ coating $\left(R^{2}=0.970\right) ; \nabla: 15 \%$ w/w coating $\left(R^{2}=0.92\right) ; \diamond: 20 \%$ w/w coating $\left(R^{2}=0.88\right)(\mathrm{B})$.

Figure 7, release profiles for the $20 \% \mathrm{w} / \mathrm{w}$ coating level, where the ionic concentration in the medium was reduced 10- and 100-fold, are depicted for particle (Fig. 7A) and film (Fig. 7B) diffusion controlled models. In both cases, the particle diffusion controlled process still gives a better fit to the linear curve than the film diffusion model. The fit quality was somewhat poor and this was due to the burst effect observed at the beginning of the release process. These ionic concentrations are
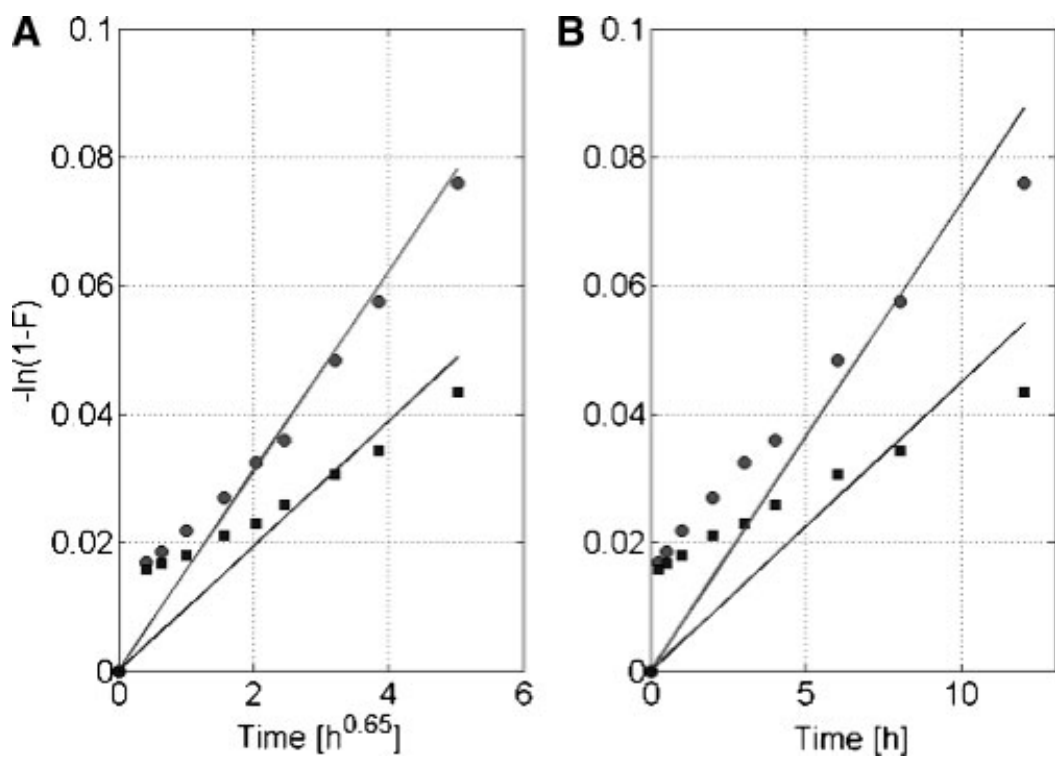

Figure 7. DM release profiles from $20 \% \mathrm{w} / \mathrm{w}$ coated pellets at different ionic concentration of the release medium plugged into particle diffusion controlled model (O: $0.01 \mathrm{~N} \mathrm{HCl}, R^{2}=0.942$ and $\square$ : $0.001 \mathrm{~N} \mathrm{HCl}, R^{2}=0.673$ ) (A); and film diffusion controlled model (B) (O: $0.01 \mathrm{~N} \mathrm{HCl}, R^{2}=0.743$ and $\square: 0.001 \mathrm{~N} \mathrm{HCl}, R^{2}=0.176$ ). 
far less than those encountered in a gastric environment. From all the foregoing findings, the film diffusion controlled process is insignificant in the present enquiry. Hence, for the development of the mechanistic model it is plausible to consider only the particle diffusion as a rate controlling mechanism.

\section{Mechanistic Model}

It is apparent that the use of the approximate models is limited to the identification of the rate controlling mechanism and do not help to conduct further study with respect to other processes such as swelling and those that are related to the design of the dosage forms. As a result, it is deemed necessary to develop more detailed models for such applications. Swelling was considered in the development of the detailed numerical model. Experimental swelling kinetics studies show that 16 and $8 \% \mathrm{v} / \mathrm{v}$ swelling was attained in equilibrium with the uncoated and coated pellets, respectively. The swelling kinetics was extracted from the experimental results and was used in the model to study the effect of the swelling on the DM release profile. Moreover, the dependence of the diffusion coefficient on the swelling was obtained by calculating the diffusion coefficients from each measured time step of the drug release from the uncoated pellets. The obtained diffusion coefficients were fitted to Eq. 16 and the constant $\beta$ was found to be -0.31 . The small value of $\beta$ indicates that indeed the effect of the swelling on the coefficient of diffusion is small. This constant was also used for the counter ion and water diffusion dependence on swelling, with the measured values as the diffusivities at the equilibrium swollen state. In Figure 8A the comparison between simulated swelling and experimental results is shown. It is worthwhile to emphasize that the pellets used were drug loaded and the resin when not loaded with drug swells to about $33 \% \mathrm{v} / \mathrm{v}$. The swelling kinetics of the pellets was well captured by the model (Fig. 8A) as can be confirmed from the fit between the two curves visually. In Figure 8B, simulation results with and without the swelling as well as with the experimental data are compared. To simulate the phenomenon where swelling was ignored two diffusion coefficient values were used, namely the values before and after swelling. It can be observed that (Fig. 8B) the swelling does not have substantial effect on the drug release profile. Besides the small swelling level of the resinate complex, the swelling kinetics takes place faster (in the first 15-30 min
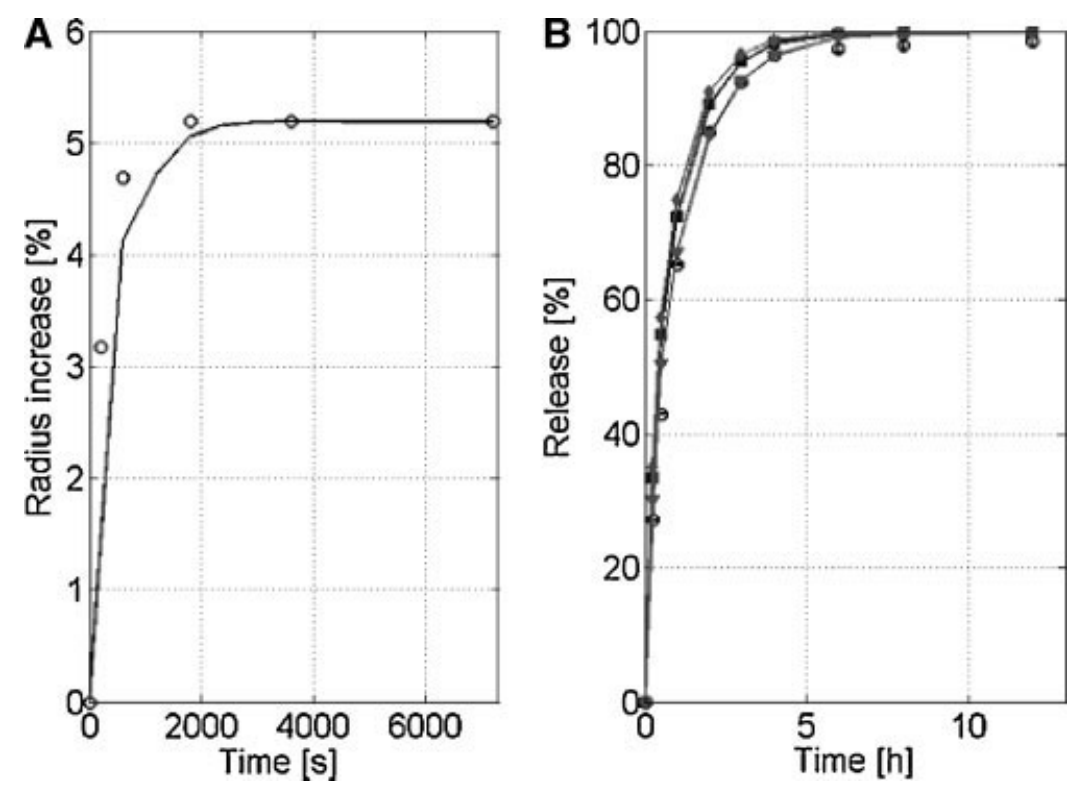

Figure 8. Comparison between simulated and experimental swelling kinetics for the uncoated pellets expressed as the percentage increase in radius. $\bigcirc$ : experiment; simulation (A); Comparison of simulation results with and without swelling as well as with the experimental data for the uncoated pellets. $\bigcirc$ : experiment; $\longrightarrow$ - simulation with swelling; —- simulation with no swelling with diffusivity coefficient before swelling; ——: simulation with no swelling with diffusivity coefficient after swelling (B). 
where only $20-30 \%$ of release takes place) as compared to the diffusion process of the drug, which takes 3-4 h. It was observed that for the coated pellets (results not shown) the swelling takes place in the first couple of minutes and swells only to $8 \% \mathrm{v} / \mathrm{v}$ level. It should be noted that the inclusion of the swelling process in the developed model substantially complicates the modeling building as well as solving process. Thus, in order to reduce the computational cost and that the model remains useful for further studies (such as, for instance, stochastic simulation of the coating nonuniformity) the swelling module can be shut off. In subsequent investigation this approach was pursued.

\section{Model Validation}

The model was validated using experimental drug release profiles using different levels of coating including $0 \%$ (Fig. 8B), 5, 10, 15, and $20 \%$ w/w. Comparisons between the experimental and simulation results for the coated pellets are shown in Figure 9. The model has captured the drug release dynamics of both the coated and uncoated pellets, as can be visually confirmed by the fit between predictions and experimental results. To compare the observed fit quantitatively, Root Mean Square Error (RMSE) values were calculated for each case. The calculated
RMSE ranges from 1.24 to $2.44 \%$ deviation. The maximum deviation was observed for the $20 \%$ coated pellets. Due to the presence of a thicker coating, one expects an initial lag period which the model has predicted. However, the experimental results show what appears to be an initial burst phenomenon. This could be due to the existence of a fraction of uncoated surface or unwashed drug particles on the surface of the coating. This is apparent for the 5\% coated pellets (Fig. 9). The SEM (Fig. 3) and confocal (Fig. 4) images show that presence of dents on the surface of the coated pellets. Defects and holes in the coating were also observed in the confocal images. Thus, coating nonuniformity and variation in particle size have played a role on the observed discrepancy.

It has already been shown that increased predictive accuracy was achieved by considering the dynamics description of all relevant processes instead of quasi static treatment. ${ }^{12}$ The inclusion of the dynamic description of all the relevant processes and employing numerical approaches, an improved model in terms of investigation of the effect of processes usually ignored in the consideration of the simpler models was possible. The other advantage of using numerical methods, especially Finite Element approach, is the flexibility in considering other geometries than the regular shapes thus enabling investigation of shape irregularities that can substantially

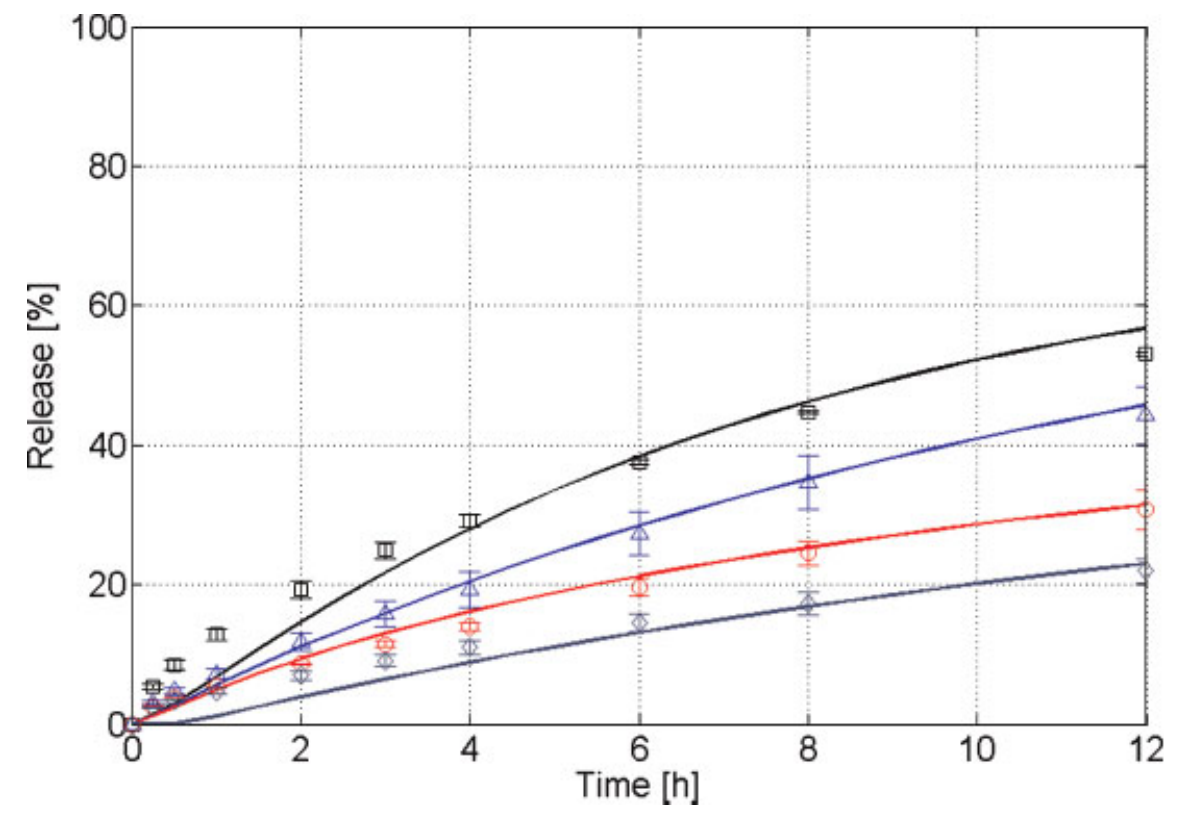

Figure 9. Simulated (solid lines) and experimental (symbols) profiles for $\square$ : $5 \% \mathrm{w} / \mathrm{w}$; $\triangle: 10 \% \mathrm{w} / \mathrm{w} ; \bigcirc: 15 \% \mathrm{w} / \mathrm{w}$; and $\diamond: 20 \% \mathrm{w} / \mathrm{w}$ coating. 
influence the attainment of the desired release profile during the design process.

\section{CONCLUSIONS}

Experimental and theoretical investigation of coated drug/ion-exchange complexes was presented. From in vitro experiments it was revealed that coating with as small as $5 \% \mathrm{w} / \mathrm{w}$ could affect the release profile significantly, indicating diffusion of the drug from the particles via the coating was the rate determining step which was further confirmed by the theoretical treatment using approximate analytical solutions of the Fick's equation (the Boyd models). Diffusion of DM through the resin matrix was the rate-limiting mechanism in drug release from the uncoated resin particles in contrast to the film diffusion. Further detailed mathematical model which considered the different domains of the coated particles (the coating and core) and pertinent processes (ionic diffusion, swelling) was developed. Swelling of the matrix for the uncoated pellets had no significant effect on the drug release profile. The developed model has captured the drug release dynamics for the coated and uncoated ion-exchange pellets. The mathematical modeling can be used as a theoretical tool to investigate drug release form coated ion-exchange complexes for design purposes.

\section{REFERENCES}

1. Lowenthal W. 1972. Mechanism of action of tablet disintegrants: Correlation of tablet mean pore diameter and porosity. J Pharm Sci 61:303304.

2. Elder DP. 2005. Pharmaceutical applications of ion-exchange resins. J Chem Educ 82:575-587.

3. Agarwal R, Mittal R, Singh A. 2000. Studies of ionexchange resin complex of chloroquine phosphate. Drug Dev Ind Pharm 26:773-776.

4. Atyabi F, Sharma HL, Mohammad HAH, Fell JT. 1996. Controlled drug release from coated floating ion exchange resin beads. J Control Release 42: 25-28.

5. Bhaskar R, Murthy RSR, Miglani BD, Viswanathan K. 1986. Novel Method to evaluate diffusion controlled release of drug from resinate. Int $\mathrm{J}$ Pharm 28:59-66.

6. Ichikawa H, Fujioka K, Adeyeye MC, Fukumori Y. 2001. Use of ion-exchange resins to prepare 100 mm-sized microcapsules with prolonged drug- release by the Wurster process. Int $\mathrm{J}$ Pharm 216: 67-76.

7. Langer DL. 1993. Polymer-controlled drug delivery systems. Acc Chem Res 26:537-542.

8. Motycka S, Nairn JG. 1979. Preparation and evaluation of microencapsulated ion-exchange resin beads. J Pharm Sci 68:211-215.

9. Borgquist P, Zackrisson G, Nilsson B, Axelsson A. 2002. Simulation and parametric study of a filmcoated controlled-release pharmaceutical. J Control Release 80:229-245.

10. Farrell S, Sirkar KK. 2001. A mathematical model of a hybrid disperssed network-membrane based controlled release system. J Control Release 70: 51-61.

11. Farrell S, Sirkar KK. 1999. A mathematical model of an acqueous-organic partition based controlled release system using microporous membranes. J Control Release 61:345-360.

12. Frenning G, Tunon A, Alderborn G. 2003. Modelling of drug release from coated granular pellets. J Control Release 92:113-123.

13. Koizumi T, Ritthidej GC, Phaechamud T. 2001.Mechanistic modeling of drug release from chitosan coated tablets. J Control Release 70: 277-284.

14. Lee DJ, Liao YC. 1995. Slow-release from a coated sphere with slightly deformed coating. J Pharm Sci 84:1366-1373.

15. Liao YC, Lee DJ. 1997. Slow-release from a coated sphere with slight deformations of coating film and drug matrix. J Pharm Sci 86:92-100.

16. Sirotti C, Colombo M, Grassi M. 2002. Modeling of drug release from poly-disperse microencupsulated spherical particles. J Microencupsulation 19: 603-614.

17. Du C, Zhou J, Shaviv A, Wang H. 2004. Mathematical model for potassium release from polymercoated fertiliser. Biosyst Eng 88:395-400.

18. Lu SM, Lee SF. 1992. Slow release of Urea through latex film. J Control Release 18:171-180.

19. Higuchi T. 1961. Rate of release of medicaments from ointment bases containing drugs in suspension. J Pharm Sci 50:874-875.

20. Korsemeyer RW, Gurny R, Doelker EM, Buri P, Peppas NA. 1983. Mechanism of solute releasefrom porous hydrophilic polymers. Int J Pharm 15: 25-30.

21. Peppas NA. 1985. Analysis of Fickian and nonFickian drug release from polymers. Pharm Acta Helv 60:110-111.

22. Boyd GE, Adamson AW, Myers LS, Jr. 1947. The exchange adsorption of ions from aqueous solutions by organic zeolites. II. Kinetics. J Am Chem Soc 69: 2836-2848.

23. Reichenberg D. 1953. Properties of ion-exchange resins in relation to their structure. III. Kinetics of exchange. J Am Chem Soc 75:589-597. 
24. Costa P, Sousa Lobo JM. 2001. Modeling and comparison of dissolution profiles. Eur J Pharm Sci 13:123-133.

25. Motycka S, Nairn JG. 1978. Influence of wax coatings on release rate of anions from ionexchange resin beads. J Pharm Sci 67:500-503.

26. Helfferich F. 1962. Ion Exchnage. editor, New York: McGraww-Hill. p 624.

27. Siepmann J, Peppas NA. 2001. Modeling of drug release from delivery systems based on hydroxy- propyl methylcellulose (HPMC). Adv Drug Deliv Rev 48:139-157.

28. Pongjanyakul T, Prakongpan S, Rungsardthong U, Chancham P, Priprem A. 2005. Characteristics and in vitro release of dextromethorphan resinates. Powder Technol 152:100106.

29. Hering B, Bliss H. 1963. Diffusion in ion exchange resins. AIChE J 9:495-503. 